\title{
P010: Bloodstream infections by drug-resistant organisms in a secondary hospital
}

\author{
T Gimenez-Julvez ${ }^{1 *}$, M Rodriguez-Aguirregabiria ${ }^{1}$, C Campelo², E Palencia-Herrejon', MJ Moreno Sanchez ${ }^{1}$, \\ $S$ de Juan-García ${ }^{1}$ \\ From 2nd International Conference on Prevention and Infection Control (ICPIC 2013) \\ Geneva, Switzerland. 25-28 June 2013
}

\section{Introduction}

Bloodstream infections (BSI) are important causes of morbidity and mortality. Most of all, when are caused by drug-resistant organisms (DR).

\section{Objectives}

To investigate the epidemiology, etiology, systemic response and treatment of DR-BSI.

\section{Methods}

A retrospective study was conducted about all BSI diagnosed in a secondary hospital during one year. The pattern resistant pathogen study was EPINE-EPPS project. Comparisons between groups were performed by means of the $X^{2}$ test for categorical variables or analysis of variances (ANOVA) for continuous variables.

\section{Results}

We included 60 patients [median and interquartile range (IQR) age, 73.5 years (60.5-79.5), 57.1\% males, median (IQR) Charlson comorbidity index, 3 (2-4), median (IQR) acute physiology and chronic health evaluation (APACHE) II score, 11 (8-15)] with 63 DR-BSI of which $71.5 \%$ were nosocomial and healthcare-associated BSI.

Unknown and intravascular catheter-related DR-BSI accounted for $49.2 \%$ of cases. Among secundary infections, the source was $37.5 \%$ urinary track, $31.2 \%$ intraabdominal and $15.6 \%$ respiratory track infections.

Overall DR-BSI, DR-Gram-positive cocci were 55.6\%. The most common isolated pathogens were staphylococcus coagulase-negative and S. aureus. Among DRGram-negative bacilli, $12.2 \%$ of enterobacteracea family produced extended-spectrum B-lactamasas. We found 5
DR-BSI caused by Acitetobacter carbapenem resistant and 3 DR-BSI by P. aeruginosa carbapenem resistant.

Median time to diagnosis for DR-Nosocomial BSI was 14 days (IQR), 7-35 days after hospital admission. For Gram-negative was 11 days (7.5-31.5) and for Grampositive 19 days (7-29).

Only $31.7 \%$ of DR-BSI received appropriate initial empirical antimicrobial therapy versus $73.5 \%$ of non DR-BSI $(\mathrm{p}<0.001)$. More than one third $(36.5 \%)$ of the episodes occur with significant systemic response (severe sepsis or septic shock). The crude mortality rate was $25.4 \%(\mathrm{p}<0.001)$. If the patient developed severe sepsis or septic shock crude mortality rose to $52.2 \%$.

\section{Conclusion}

Information about local epidemiology is important to develop prevention and control strategies in drug-resistant microorganism and to improve the management of BSI.

\section{Disclosure of interest}

None declared.

\section{Author details}

${ }^{1}$ Hospital Universitario Infanta Leonor, Spain. ${ }^{2}$ BR-Salud, Madrid, Spain.

Published: 20 June 2013

doi:10.1186/2047-2994-2-S1-P10

Cite this article as: Gimenez-Julvez et al:: P010: Bloodstream infections by drug-resistant organisms in a secondary hospital. Antimicrobial Resistance and Infection Control 2013 2(Suppl 1):P10.

${ }^{1}$ Hospital Universitario Infanta Leonor, Spain

Full list of author information is available at the end of the article 